\title{
The NASA/LRC Computerized Test System
}

\author{
W. KIRK RICHARDSON, DAVID A. WASHBURN, WILLIAM D. HOPKINS, \\ E. SUE SAVAGE-RUMBAUGH, and DUANE M. RUMBAUGH \\ Georgia State University, Atlanta, Georgia
}

\begin{abstract}
A new testing package, including apparatus and tasks, is described for the study of behavior of a variety of species in a variety of experiments. The package is described with respect to the kinds of comparative psychological investigations for which it is well suited. The preliminary data generated within this new testing paradigm demonstrate that the NASA/LRC Computerized Test System provides a flexible yet powerful environment for the investigation of behavioral and psychological processes.
\end{abstract}

The NASA/LRC Computerized Test System (NASA/ LRC-CTS) is a new test package (apparatus and tasks) initially designed to address the specific needs of the $\mathrm{Na}$ tional Aeronautics and Space Administration (NASA) for comparative and biomedical research concerning the effect of microgravity on behavioral and physiological functioning. ${ }^{1}$ The NASA/LRC-CTS has also been demonstrated to provide a rich and robust testing environment for the cognitive and neuropsychological capacities of great apes, rhesus monkeys, mentally retarded and normally developing children, and adults. Of these subject populations, the research with the rhesus monkeys (e.g., Rumbaugh, Richardson, Washburn, Savage-Rumbaugh, \& Hopkins, 1989; Washburn, Hopkins, \& Rumbaugh, 1989a, 1989b, 1990) will be used in the present instance to illustrate the utility of the testing paradigm.

The package consists of hardware (a computer and peripherals) and software (a series of programs or tasks) that allow for an organism to be trained and tested. The tasks are designed to assay many psychological capacities, including attention, learning, memory, perception, prediction, and psychomotor functioning. The features, many of which stem directly from design criteria imposed by the projects for which the package was developed, make it potentially useful to behavioral scientists who wish to automate or to initiate a program of research with humans or nonhuman primates. These design criteria are listed in Table 1.

\section{Hardware}

It was believed that a general-purpose microcomputer would best allow these criteria to be satisfied. The initial hardware platform (a Zenith 159) is an 8- $\mathrm{MHz}, 8088$ based machine with $256 \mathrm{~K}$ of RAM, one $20 \mathrm{MB}$ hard disk,

The research and development reported here were supported by Grant NAG2-438 from the National Aeronautics and Space Administration. Additional support was provided by the National Institutes of Health (NICHD 06016) and by the College of Arts and Sciences of Georgia State University. Inquiries should be sent to W. Kirk Richardson, Psychology Department, Georgia State University, Atlanta, GA 30303.
Table 1

Design Criteria of the NASA/LRC Computerized Test System

1. Flexibility

a. Multiple behaviors assayed.

b. Multiple reinforcers (edibles, secondary reinforcers, noningestible rewards such as video).

c. Visual or auditory stimuli.

2. Growth and Development

a. Integration of new hardware and technology.

b. Integration of new tasks and software

3. Interexperiment compatibility

a. Movement between tasks without significant retraining of subjects.

b. Control over parameters of each task.

c. Potential for timely introduction of new experiments or tasks

4. Behavioral productivity

a. Maintain a high level of behavioral output for several hours per day and for several days in sequence.

b. Motivational, in that a qualitatively high level of performance is obtained for each subject.

c. Little or no deprivation and no use of aversive or punitive stimuli.

5. Economical

a. Hardware and software must be made available in several labs.

b. Not labor-intensive, in that extensive experimenter-subject interaction is not required.

6. Technology transfer

a. "User-friendly" even to personnel not familiar with psychology or computer operations.

b. Functional and supported at other laboratories despite disparate locations or interests.

and one $360 \mathrm{~K}$ floppy disk drive. The monitor is a 32.5$\mathrm{cm}$ diagonal color display driven by a CGA color graphics board. A standard, spring-loaded analog joystick, connected to a standard IBM game port mounted in one of the expansion slots of the computer, serves as the general input device. A 12-channel digital I/O board (MetraByte PIO-12), installed in a second expansion slot, provides interfacing for up to eight reward devices. A second MetraByte board (CTM-05) is used by some applications for millisecond timing. Sound generated by the computer is projected through an external speaker/amplifier (Radio Shack 32-2031), which replaced the computer's speaker. ${ }^{2}$ This speaker has volume control, allowing adjustment as needed. 
Joysticks are excellent general-purpose response devices for research with chimpanzees (Savage-Rumbaugh, 1986) and with humans, and thus they were selected for use in the NASA/LRC-CTS. However, joystick technology had not been used for complex tasks with rhesus monkeys prior to the development of the NASA/LRC-CTS. Indeed there was good reason to doubt that the rhesus monkey could learn to use the joystick (Meyer, Treichler, \& Meyer, 1965; Stollnitz, 1965; cf. Rumbaugh et al., 1989).

Each rhesus' test station consisted of the color monitor and speaker mounted with a Lexan enclosure. The joystick base was mounted behind the Lexan, with the stick protruding outward horizontally through a hole. Only the handle of the joystick was exposed to the subject; wires and equipment were protected by the Lexan. The stick was perpendicular to and $20 \mathrm{~cm}$ below the monitor. The stick was positioned in this orientation so that the movements of the joystick would be isomorphic to (i.e., in the same plane and direction as) movements of the image on the screen controlled by the joystick (the cursor).

The monkeys were individually tested in their home cages. The cages were rolled to the front of the test stations and fixed in place, $20 \mathrm{~cm}$ from the Lexan enclosure, during testing. Thus, each subject could reach through the mesh of the cage and manipulate the joystick while looking at the monitor. Food rewards were delivered to pellet cups mounted on the sides of each cage.

\section{Software}

All tasks and utilities are written in QuickBasic (version 4.5) and are compiled with the Microsoft BASIC Compiler (version 6.0).

\section{CHASE-THE BASE TASK}

The training system is designed around a base task, which all subjects must master. Other tasks are designed to be easy behavioral transitions from this base task. The base task requires that subjects use the joystick to control the movement of a small, computer-generated image (called the cursor) on the color monitor. In order for the subject to receive reward, the cursor has to be guided to a collision with a target (another computer-generated image). This basic task (CHASE) has behavioral components that are easily expandable to other tasks. Once the animal has learned about the test environment (the joystick, the availability of food, the relation between joystick movements and cursor movements), there is rapid transfer to other tasks involving these same components.

CHASE is a trial-based procedure. The beginning of a trial is signaled by the presentation of the cursor and the target on the monitor. The initial position of the cursor is at the center of the screen, while the initial position of the target varies randomly from trial to trial. Both cursor and target remain stationary until the joystick is manipulated. At that time, the cursor moves in the direction isomorphic to the angle of joystick deflection while the target moves along a preselected course (e.g., a straight line, a circle, randomly, etc.). As the cursor moves, sound feedback is presented through the speaker. Both the cursor and target move until one of three events occurs: (1) The cursor reaches the border of the screen; (2) the joystick is released or returned to the center position; (3) the cursor and the target collide. When the cursor hits a side of the screen, or, alternatively, if the joystick is released or returned to the center position, all movement is stopped until the joystick is again used to move the cursor (for instance, away from the side of the screen). A cursor-target collision results in sound feedback and, if scheduled, in presentation of a reward. After a variable intertrial interval, the next trial is presented. During the intertrial interval, the screen is blank except for the number of the next trial, which is displayed in the upper left corner for the researcher's benefit. This cycle continues until a preset number of trials is delivered or until the F1 key is pressed. The F1 key allows the user to terminate in an orderly manner a session prior to delivery of the scheduled number of trials and to return immediately to a menu.

\section{Pretraining for CHASE}

To train the subject in the precursor behaviors for the mastery of CHASE, a progression of other tasks was designed. The general training format first provides an easy format for the subject to manipulate the joystick and receive a reinforcer; subsequently, the tasks become systematically more difficult, requiring increasing skill in joystick manipulation. In this way, one capitalizes on the natural curiosity that typically surrounds the introduction of a joystick. An autoshaping task, which will automatically shape an animal to manipulate the joystick, is also available in the event that an animal fails to display an interest in the joystick. Each of these tasks, and data supporting their effectiveness, is discussed in detail by Rumbaugh et al. (1989).

Initially, each side has a colored box covering its full length and thus serves as a target (the SIDE task). Essentially, the screen shows the cursor surrounded by targets. The subject is required to move the cursor into contact with any target wall to receive a reward. Somewhat like shooting a gun inside a house, the moving cursor eventually has to hit something. As the monkey comes to move the joystick reliably, the number of sides of the screen that are active as targets can be reduced from four to three, to two, and finally to only one. During this reduction, the appropriate number of target walls is randomly selected and assigned. Thus, the subject must increasingly come to discern the loci of targets from nontarget areas. Next, the size of the remaining target wall is reduced in three steps until the target is but a small box. Each reduction is made when the animal is performing well. If, after a transition, the subject makes many errors, the task is returned to the previous phase for continued training. Thereby the subject is increasingly required to move the joystick with precision in order to obtain rewards most efficiently. 
When the subject reliably guides the cursor to the small, stationary target, the task can be changed so that the target moves. This is the CHASE task at its most basic level. Our subjects learned these tasks with amazing speed and facility, and they engage in task-related behavior many hours a day, even though they are neither food- nor fluiddeprived.

\section{Unique Program Features}

Picture libraries. A picture library is built into the programs, to provide a limitless supply of pictures to be used as cursors and targets. Both the cursor and the target are selected from the library. Within certain constants, stimuli can be any shape, size, and color supported by the computer. Each library page is a bit-mapped graphics screen (BSAVE format). Consequently, a researcher may make additional stimuli by using any package, such as PCPG or PC Storyboard, that allows pictures to be created as or changed to a bit-map file.

Stimulus movement. Stimuli need not remain static on screen, but can move along various paths at various speeds. Data collected under conditions in which the stimuli moved (Washburn et al., 1989b) indicate that learning may be amplified under these conditions.

Parametric control. CHASE has many parameters that can be controlled by the researcher through menu selections. Through manipulation of these parameters, a variety of behaviors and corresponding psychological operations can be examined. A list of these parameters is presented in the Appendix.

Data collection. The programs allow for collection of an array of data. In addition to accuracy and response latency, the actual path of responding can be recorded. Because the appearance of movement is accomplished by programming a series of still screens with the "moving" object being erased and re-presented in a slightly different position at each step in the movement sequence, the exact sequence and speed of target and cursor locations can be recorded as a series of $x$ - and $y$-coordinates and clock readings. These data can then be replayed for later viewing and/or analysis. The data can be sampled and analyzed or replayed.

\section{FINAL PACKAGE}

The package is designed to be easy to use, to allow easy selection of parameters, and to be supported by the developers. Several steps were taken in the design of the software to make this possible. All program selections are made from menus. Unallowable values are trapped by the program, and the user is notified to enter another, acceptable value. Each time a program is executed, the parameters from the last session are loaded as default values, so that, for most sessions, only the session number has to be changed.

To date, several tasks have been implemented in the NASA/LRC-CTS, each with an individual set of adjusta-
Table 2

A List of Tasks and Descriptions

\begin{tabular}{|c|c|}
\hline AUTOSHAP & $\begin{array}{l}\text { Pellet delivered at various times to "magazine } \\
\text { train" and shape each animal. }\end{array}$ \\
\hline SIDE & Move the cursor to 1-4 target walls. \\
\hline CHASE & Chase a moving target. \\
\hline PURSUIT & Catch and track a moving target. \\
\hline LASER & "Shoot" at moving targets from a stationary turret \\
\hline COMPETE & $\begin{array}{l}\text { Both animals shoot at the same target, such that } \\
\text { each competes for the reward. }\end{array}$ \\
\hline DL & Six-trial discrimination learning set problems. \\
\hline $\mathrm{TI}$ & $\begin{array}{l}\text { Transfer index automatically measured at } 67 \% \text { or } \\
84 \% \text { criterion levels. }\end{array}$ \\
\hline MTS & Matching to sample (with two choice stimuli). \\
\hline SELECT & $\begin{array}{l}\text { Choose the task in which to engage, and receive } \\
\text { five trials on the selected task. }\end{array}$ \\
\hline STM & Delayed MTS. \\
\hline HOLE & Chase with a "black hole." \\
\hline SDI & $\begin{array}{l}\text { Series of same/different training and testing } \\
\text { programs. }\end{array}$ \\
\hline
\end{tabular}

ble parameters. Each of these tasks represents a variation the base task CHASE. The tasks are listed in Table 2.

Two manuals have also been prepared: a program manual and a training manual. The program manual explains how to set up the system and use the programs. The training manual is a short course in animal training that explains the purpose of procedures such as magazine training and shaping and provides guidelines for advancing a subject from one phase to the next phase of training.

\section{BEHAVIORAL RESULTS}

Again, the NASA/LRC-CTS has been productively employed in research with a wide variety of subject populations. For example, several laboratories in addition to the Language Research Center have now successfully used this package to train monkeys on the CHASE task. The training generally proceeds quickly, and the animals perform many trials a day (300-1,200, depending on familiarity and task demands, are typical). Little direct human intervention is needed to train the monkeys. The monkeys do not have to be habituated specifically to the presence of humans prior to training, and they do not have to be reduced in body weight or otherwise deprived to obtain high levels of performance. It was necessary neither to chair or otherwise restrain the animals nor to prohibit the animals from looking at the joystick at any time during training or testing.

There has been excellent transfer of learning each time changes have been made in the apparatus. For example, rotating the joystick $90^{\circ}$ so that it pointed up (vertical) rather than out at the monkey (horizontal) had a negligible and transient effect on the behavior of 2 experienced monkeys. Similar results were observed when the size of the joystick was changed from large to small, the size of the monitor was reduced, and sound feedback was removed. The monkeys seem to be relatively unaffected by changes in the physical characteristics of the apparatus, at least once the task-appropriate behaviors have been 
mastered. We believe that positioning the monitor directly before the animals, sound and cursor movement contingent on and isomorphic to joystick manipulation, and timely and reliable pairing of behavior with visual results and subsequent reward have contributed to the robustness of these behaviors. Subsequent study with additional animals will determine the degree to which such characteristics affect initial learning.

New tasks have proven easy to introduce to monkeys experienced with the CHASE task. A modification of the CHASE task to require that the monkey maintain contact between the cursor and the target (PURSUIT) was learned without disruption. The presentation of two targets (one positive and one negative) in a simultaneous discrimination learning paradigm was quickly accepted and mastered by our monkeys, as were additional stimuli within a matching-to-sample paradigm (Washburn et al., 1989b). Even changing the task so that manipulating the joystick sent a beam of light out of a turret, with the requirement that the light beam had to hit a moving target (the LASER task), did not deter our monkeys (Washburn et al., 1989b). To date, a wide variety of developmental tasks has been implemented successfully to train or test the monkeys' learning, discrimination, short- and long-term memory, attention, psychomotor coordination, and capacity for prediction. The animals have readily discovered the contingencies each time a new task was introduced.

Other applications can easily be envisioned. A subject's leg could be exercised by making the joystick available to a foot. A force-measuring joystick could be used to track the decay of muscle strength as a function of time in microgravity. Integration of devices for the tracking of eye movements and other input peripherals are also anticipated.

Finally, we should note that these animals worked many hours a day, performing hundreds of trials for food reward when they were not deprived of food. Each night, the monkeys were given more food than they ate during the night. The next morning, the excess food was removed and a task was made available. The animals readily started to work even when the reward was a small (45- or 97$\mathrm{mg}$ ) pellet of chow very similar in composition to the chow just removed from the cage. Our suspicion is that the tasks have some motivating or rewarding value of their ownwhich itself is an interesting subject of current experimentation.

\section{SUMMARY}

We believe that this testing package offers new ways to study the questions of psychology efficiently, appropriately, and economically, particularly with young children and nonhuman primates. The paradigm allows the study of more complex behaviors than is possible with a simple bar in an operant chamber or with lights or objects as stimuli, as in variations of the Wisconsin General Test Apparatus (WGTA). In addition to this potential for measuring more complex behaviors, there is evidence to suggest that even behavior that can be measured within traditional testing paradigms, such as, for example, discrimination learning sets and transfer indexes, benefit from this new testing procedure (Washburn et al., 1989a, 1989b).

The testing package also offers a direct benefit for comparative psychological research in that subjects from various populations can be tested under comparable conditions. That is, the same task, with the same parameters and apparatus, can be used across species or subject populations. In this way, control is increased over testing conditions that have typically been problematic as experimental confounds.

Finally, this test environment may also provide primate colonies with the basis for enrichment programs that will enhance the behavioral environment for animals maintained in research colonies and in zoos. It seems reasonable to suggest that, in this way, the test system described here may contribute to better treatment-as well as better understanding- of several subject populations.

\section{REFERENCES}

Meyer, D. R., Treichler, F. R., \& Meyer, P. M. (1965). Discretetrial training techniques and stimulus variables. In A. M. Schrier, H. F. Harlow, \& F. Stollnitz (Eds.), Behavior of nonhuman primates: Modern research trends. (Vol. 1, pp. 1-49). New York: Academic Press.

Rumbaugh, D. M., Richardson, W. K., Washburn, D. A., SavageRumbaugh, E. S., \& HoPKINS, W. D. (1989). Rhesus monkeys (Macaca mulatta), video tasks, and implications for stimulus-response spatial contiguity. Journal of Comparative Psychology, 103, 32-38.

Savage-Rumbaugh, E. S. (1986). Ape language: From conditioned response to symbol. New York: Columbia University Press.

Stollnitz, F. (1965). Spatial variables, observing responses, and discrimination learning sets. Psychological Review, 72, 247-261.

Washburn, D. A., Hopkins, W. D., \& Rumbaugh, D. M. (1989a). Automation of learning-set testing: The video-task paradigm. Behavior Research Methods, Instruments, \& Computers, 21, 281-284.

Washburn, D. A., Hopkins, W. D., \& Rumbaugh, D. M. (1989b). Video-task assessment of learning and memory in macaques (Macaca mulatta): Effects of stimulus movement upon performance. Journal of Experimental Psychology: Animal Behavior Processes, 15, 413-420. Washburn, D. A., Hopkins, W. D., \& Rumbaugh, D. M. (in press). The effects of competition on video-task performance in monkeys (Macaca mulatta). Journal of Comparative Psychology.

\section{NOTES}

1. The LRC Computerized Test System programs are available to interested colleagues. For more information on the system and its availability, write W. Kirk Richardson, Psychology Department, Georgia State University, Atlanta, GA 30303.

2. Since the computers are not equipped with an external speaker jack, one must be made for this application. This is accomplished by opening the computer case to reveal the internal speaker; disconnecting the wires going to the internal speaker; and connecting these lines, via a length of two-conductor, insulated wire, to the microphone jack of the external speaker/amplifier. This procedure is both easy and, we believe, very important for the training of animals, in that it permits sound feedback to be produced from a site near the visual consequences of joystick manipulation (i.e., the monitor). 


\section{APPENDIX \\ Parameters of the CHASE Task}

I Subject's name and/or number

2. Trial parameters

a. Truals per session

b. Intertrial interval

c Drop-out tıme

3. Data parameters

a Keep detailed data? (Yes/No)

b. Disk drive for detailed data

c Density of detalled data

d. Disk drive for summary data

4. DRO size

5. Fixed ratio size

6. Reward lines used

7. Sprite parameters
a. Speed
b. Picture
c. Collision distance
d. Collision duration
e. Joystıck dead zone

8. Target parameters
a. Picture
b. Path shape
c. Path parameters

9 Sound with movement? (Yes/No)

Key

2 c Drop-out time: The interval after which a trial will be automatically termınated if the joystick is not manipulated The drop-out contingency does not become operative during a trial until the stick has been moved out of the center at least once.

3 a. The detailed data. The pixel-by-pixel recording of response path

$7 \mathrm{c}$. Collision distance: The maxımum distance between the cursor and a target for a "collision" or contact to be registered.

7.d. Collision duration. The interval of continuous contact that must be maintained between a cursor and target to terminate a trial.

7 e Dead zone: The degree to which a joystick must be displaced to initiate cursor movement.

8.b. Target path: The path followed by the target may be a rectangle, circle, line, sawtooth, or a random pattern.

$8 \mathrm{c}$. Path parameters: Parameters of each path (such as the radius of the circle) may be set by the researcher. 